\title{
L'ASSURANCE DE LA PROPRIÉTÉ DE L'ÉTAT DANS LA RÉPUBLIQUE POPULAIRE DE POLOGNE
}

\author{
Dr. Antoni Banasinski \\ Pologne
}

I. Le fait que le 8-e Colloque ASTIN a lieu en Pologne donne une bonne occasion pour informer les membres de l'ASTIN sur l'organisation actuelle des assurances dans ce pays qui est d'ailleurs pareille à celle des assurances dans les autres pays socialistes. Dans le présent rapport sera présenté en particulier le problème des assurances du patrimoine national (propriété de l'État) qui comprennent aussi les risques d'assurance importants et complexes.

2. Après la II-e Guerre Mondiale la Pologne a connu de profonds changements sociaux et économiques. La grande et moyenne industries ont été nationalisées. On a réalisé la réforme agraire. Le transport et l'échange des marchandises ont été confiés à des entreprises d'Etat et coopératives. Tout le système financier, y compris les banques et les assurances, a été nationalisé, etc. Actuellement en Pologne les activités d'assurance sont effectuées par deux établissements.

Państwowy Zakład Ubezpieczeń -- en abréviation PZU - (Assurance Nationale Polonaise);

Towarzystwo Ubezpieczeń i Reasekuracji „WARTA” — en abréviation ,WARTA" (Compagnie d'Assurances et de Réassurances SA ,WARTA").

3. La PZU ne s'occupe que des assurances du pays, c.à.d. des assurances des objets situés en Pologne et des personnes domiciliées en Pologne.

La ,WARTA" s'occupe de toutes assurances extérieures liées aux paiements en devises (contractées pour la plupart en monnaie étrangère), ainsi donc avant tout des assurances des marchandises dans le transport maritime, des assurances des moyens de transport maritime et fluvial, des assurances d'aviation, des assurances des véhicules automobiles etc. 
La „WARTA” développe en outre des activités de réassurance active et passive. En pratique ne sont réassurés que les objets assurés directement par la „WARTA”.

4. Les assurances du pays, entre les mains de la PZU, sont réalisées sous la forme obligatoire ou facultative.

Sont soumis à l'assurance obligatoire:

(I) les exploitations agricoles appartenant à des individus (propriété privée) et des coopératives;

(2) les propriétaires (usagers) des véhicules à moteur.

Dans les exploitations agricoles individuelles, dont on compte en Pologne plus de 3 millions, les assurances de tous risques couvrent presque toute leur propriété:

(a) les bâtiments, le mobilier, le cheptel, les produits agricoles, etc. contre l'incendie, l'inondation, les ouragans et autres événements fortuits,

(b) les cultures principales contre la grêle et l'inondation,

(c) le bétail (chevaux et bovins et dans certaines régions également les porcins) contre la crevaison.

Les assurances obligatoires des véhicules à moteur (dénommées assurances de transport) comprennent la responsabilité civile des possesseurs des véhicules ainsi que les suites des accidents arrivés à des personnes (conducteur, passagers, piétons) à la suite du trafic de ces véhicules.

Les autres assurances de biens sont contractées facultativement.

5. Les assurances du patrimoine des entreprises de l'État et des coopératives sont aussi facultatives. Les unités économiques (entreprises, coopératives) y agissent cependant suivant les recommandations ou ordres de leurs autorités supérieures (ministères, associations etc.).

En pratique les assurances contre l'incendie et les autres événements fortuits couvrent à présent tout le patrimoine des entreprises de la grande et moyenne industrie d'État ainsi que le patrimoine des coopératives. 
Il existe en outre ,l'obligation administrative" d'assurer les biens des unités économiques d'État et coopératives contre les risques du transport.

Les autres genres des assurances du patrimoine d'État et des coopératives (les assurances auto - casco, responsabilité civile, contre le vol etc.) ne sont pas aussi répandues, puisqu'elles sont contractées selon le bon gré de l'unité économique donnée.

6. L'introduction de la généralité des assurances du patrimoine socialisé (national), en disposition des entreprises particulières, d'État ou coopératives, a eu lieu à la suite de longues années d'études et de discussions théoriques et sous l'influence des besoins nés de la pratique de la gestion des entreprises d'État.

D'après certaines opinions extrémistes sur ce problème il est superflu d'assurer le patrimoine socialisé, puisqu'il n'existe qu'un seul propriétaire de ce patrimoine (l'État).

Il ne peut donc y être question d'une ,répartition des risques" entre plusieurs unités, menacées par un danger commun. Les dommages fortuits peuvent être dans ces cas compensés en dehors des assurances par le moyen de dotations convenables à partir des réserves générales de l'État (du budget).

Le point de vue opposé à ces opinions part du principe que dans le régime socialiste aussi les entreprises particulières disposent d'une grande autonomie économique, et assument une pleine responsabilité pour les biens qui leur sont confiés. Les résultats de leur activité économique sont évalués selon les principes de l'ainsi nommé calcul économique, ce principe devant prendre également en égard les dépenses destinées à la protection directe ou d'assurance des biens qui se trouvent en leur disposition.

En outre, la pratique a démontré que la compensation des dommages fortuits par le moyen des assurances est bien plus efficace qu'à partir des dotations budgétaires.

En fin de compte c'est ce deuxième point de vue qui l'a remporté. Ainsi la Pologne est un pays socialiste dans lequel la protection d'assurance s'étend en large mesure sur le patrimoine de l'État.

7. L'assurance de la propriété de l'État et des coopératives est réalisée en Pologne d'une manière simplifiée et adaptée en même 
temps aux besoins des unités économiques socialisées. Ainsi par exemple les assurances sont contractées dans le cadre des contrats généraux, sans nécessité de dresser des polices individuelles. Les sommes d'assurance des biens durables ne constituent pas lá limite de la responsabilité de l'établissement des assurances et le montant de l'indemnité est fixé selon la valeur réelle de l'objet lésé à l'état neuf, la prime est calculée par une „méthode intégrale”, qui englobe tous les risques (dangers) relatifs à l'entreprise donnée etc.

La ,méthode intégrale" du calcul de la prime part du principe que la "structure d'assurance" de l'entreprise donnée en une longue période de temps ne subit de changements majeurs. En conséquence la prime moyenne pour tous les genres des risques (incendie, explosion, inondation, avarie des machines, auto - casco, responsabilité civile etc.), calculée pour une unité de la valeur de la production annuelle de cette entreprise reste inchangée. En multipliant le taux de prime ainsi établi par la valeur du volume de la production de l'entreprise au cours de l'année donnée nous obtenons la prime annuelle pour l'assurance de l'entreprise donnée.

Il est opportun de remarquer que le taux de la prime, établi de la manière sus-dite, s'ajoute aux frais par unité de production à titre de l'assurance de celle-ci.

Il est évident qu'en cas de l'extension de la protection d'assurance d'une modification essentielle des conditions de l'assurance ou d'un changement de la structure de l'entreprise on procède à une correction appropriée du taux de la prime.

Les méthodes d'assurance de ce genre, simplifiées et en même temps différentes des méthodes traditionnelles donnent lieu à de nouveaux problèmes actuariels. Il s'agit entre autres d'établir, en partant des informations relativement modiques, une prime nette, dont le montant serait aussi proche que possible de la somme des indemnités réelles. On ne prévoit pas dans ces calculs aucun supplément destiné à créer un profit.

8. L'assurance de patrimoine d'État donne encore naissance à d'autres problèmes actuariels difficiles.

Le portefeuille des assurances du patrimoine de l'État n'est homogène ni du point de vue de la valeur des risques particuliers, ni de celui du degré auquel le patrimoine est menacé par les dangers 
soumis à la protection d'assurance. A côté de maints risques pour lesquels l'indemnité maxime ne peut pas dépasser le montant d'un million de zlotys, il existe des risques pour lesquels, dans une situation exceptionnellement catastrophique et en concurrence de circonstances défavorables, le dommage peut arriver jusqu'à un milliard de zlotys, donc presque autant que la prime annuelle dans le groupe d'assurance donné.

Dans la période après la II-e Guerre Mondiale il n'y a pas eu en Pologne de dommages de ce genre aussi grands et catastrophiques, quoique possibles en théorie. Il n'est donc pas possible d'estimer empiriquement la probabilité de leur arrivée. On ne peut que supposer que cette probabilité est infime et qu'ils sont ,pratiquement" impossibles.

9. Quant aux dommages moyens et peu importants on en observe une grande stabilité dans l'énorme portefeuille de la PZU. C'en est là, sans aucun doute, un des plus grands avantages de nature technique d'assurance, que l'on ait atteint grâce à la nationalisation des activités d'assurance et à l'organisation d'un seul établissement des assurances du pays.

Commençons par quelques exemples de la statistique du domaine des assurances obligatoires contre l'incendie de bâtiments dans environ 3 millions d'exploitations agricoles, donc relatifs à un portefeuille bien homogène. Le taux de dommages (incendies), qui exprime le rapport du montant des dommages à la valeur des objets assurés (et en même temps le rapport des indemnités à la somme d'assurance) en Pologne, dans les années r 956 - I967 se présente comme suit (en pro mille):

$$
\begin{array}{llllll}
\mathrm{I}, \mathrm{OO} & \mathrm{I}, 25 & 0,89 & \mathrm{I}, 50 & 0,3 \mathrm{I} & \mathrm{I}, 24 \\
\text { 0,9I } & \mathrm{I}, 06 & 0,98 & 0,75 & 0,89 & 0,9 \mathrm{I}
\end{array}
$$

Les éléments (facteurs) du taux de dommages présentent encore plus de stabilité. Ainsi la fréquence des évènements fortuits, dans le cas donné le nombre des incendies pour rooo bâtiments assurés, au cours de la même période de temps, était la suivante:

$$
\begin{array}{llllll}
\text { I,O8 } & \text { I,30 } & \text { I,O5 } & \text { I,4I } & \text { I,07 } & \text { I,36 } \\
\text { I,II } & \text { I,27 } & \text { I,I5 } & 0,98 & \text { I,II } & \text { I,25 }
\end{array}
$$


L'envergure des incendies, c.à.d. le rapport du nombre des bâtiments détruits au nombre des incendies s'est chiffrée à:

$$
\begin{array}{llllll}
2, \mathrm{O} 2 & 2, \mathrm{OI} & \mathrm{I}, 8 \mathrm{O} & 2,27 & \mathrm{I}, 9 \mathrm{I} & \mathrm{I}, 97 \\
\mathrm{I}, 8 \mathrm{I} & \mathrm{I}, 88 & \mathrm{I}, 95 & \mathrm{I}, 8 \mathrm{I} & \mathrm{I}, 89 & \mathrm{I}, 79
\end{array}
$$

Par contre l'intensité des dommages c.à.d. le rapport de la somme du dommage à la valeur des bâtiments lésés se montait à:

$$
\begin{array}{llllll}
0,5 \mathrm{I} & 0,52 & 0,49 & 0,55 & 0,5 \mathrm{I} & 0,54 \\
0,52 & 0,48 & 0,50 & 0,47 & 0,48 & 0,46
\end{array}
$$

Une stabilité pareille est à observer dans le portefeuille bien moins homogène des assurances du patrimoine des entreprises d'État et des coopératives contre l'incendie et les autres événements fortuits.

Dans les années I958-1967 le taux de dommages se présentait en pro mille comme suit:

$$
\begin{array}{lllll}
0,5 & 0,4 & 0,5 & 0,5 & 0,3 \\
0,3 & 0,3 & 0,4 & 0,4 & 0,4
\end{array}
$$

Il en est de même dans les assurances de transport où le taux de dommages net (compte tenu des recours à intenter contre les transporteurs), se chiffrait à:

$$
\begin{array}{lllll}
\text { 0,OI3 } & \text { 0,OII } & 0,010 & 0,0 I 2 & 0,0 I 4 \\
\text { 0,OII } & 0, \text { OII } & 0,0 I I & 0,0 I 3 & 0,0 I 3
\end{array}
$$

Les données statistiques de ce genre permettent de constater que les résultats des calculs actuariels, relatifs au montant prévu des indemnités ainsi qu'aux taux de primes nets garantissent en plein que les opérations financières de l'établissement d'assurances, se maintiendront au futur en balance.

IO. De toutes ces considérations et exemples on peut tirer la conclusion suivante:

Les grands établissements d'assurance, tels que le sont les établissements nationaux d'assurance dans les pays socialistes peuvent être sûrs de maintenir la balance de leurs finances même sans faire appel à la réassurance et sans former de réserves de garantie plus 
24 L'ASSURANCE PROpriété DE L'État EN POLOGNE

importantes. Cette balance ne peut être menacée que par un dommage aux dimensions d'une catastrophe, ce qui est possible mais fort peu vraisemblable. Comme de tels dommages peuvent ne pas arriver du tout au cours des dizaines d'années, il n'est pas possible d'en tenir compte lors des calculs actuariels normaux. 